
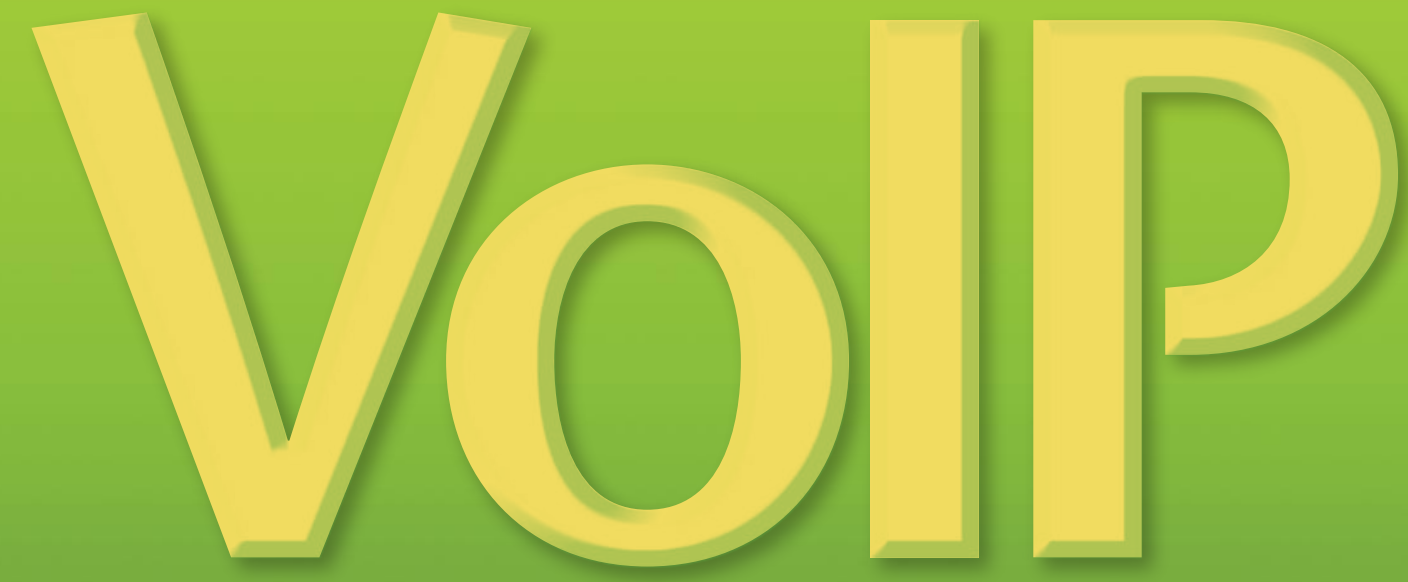

\title{
What Is it Good for?
}

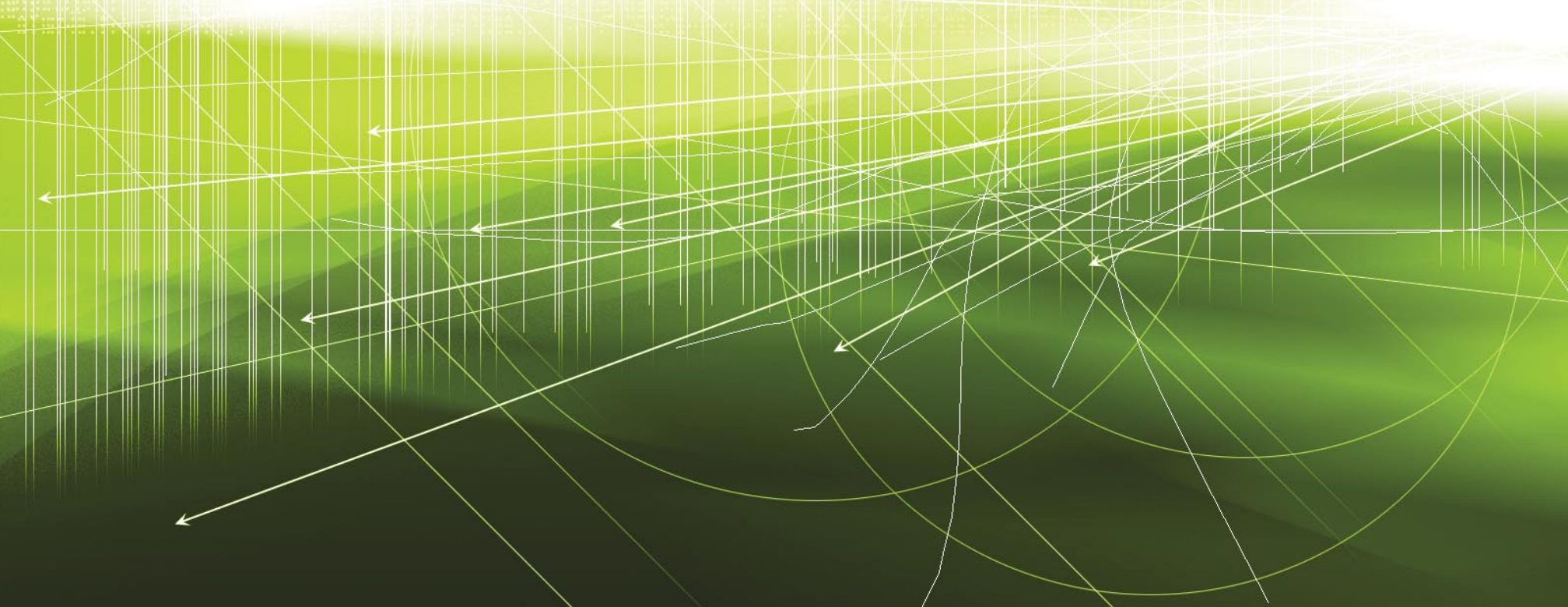

SUDHIR R. AHUIA AND I. ROBERT ENSOR, BELLLABS/LUCENT TECHNOLOGIES 


\section{Q}

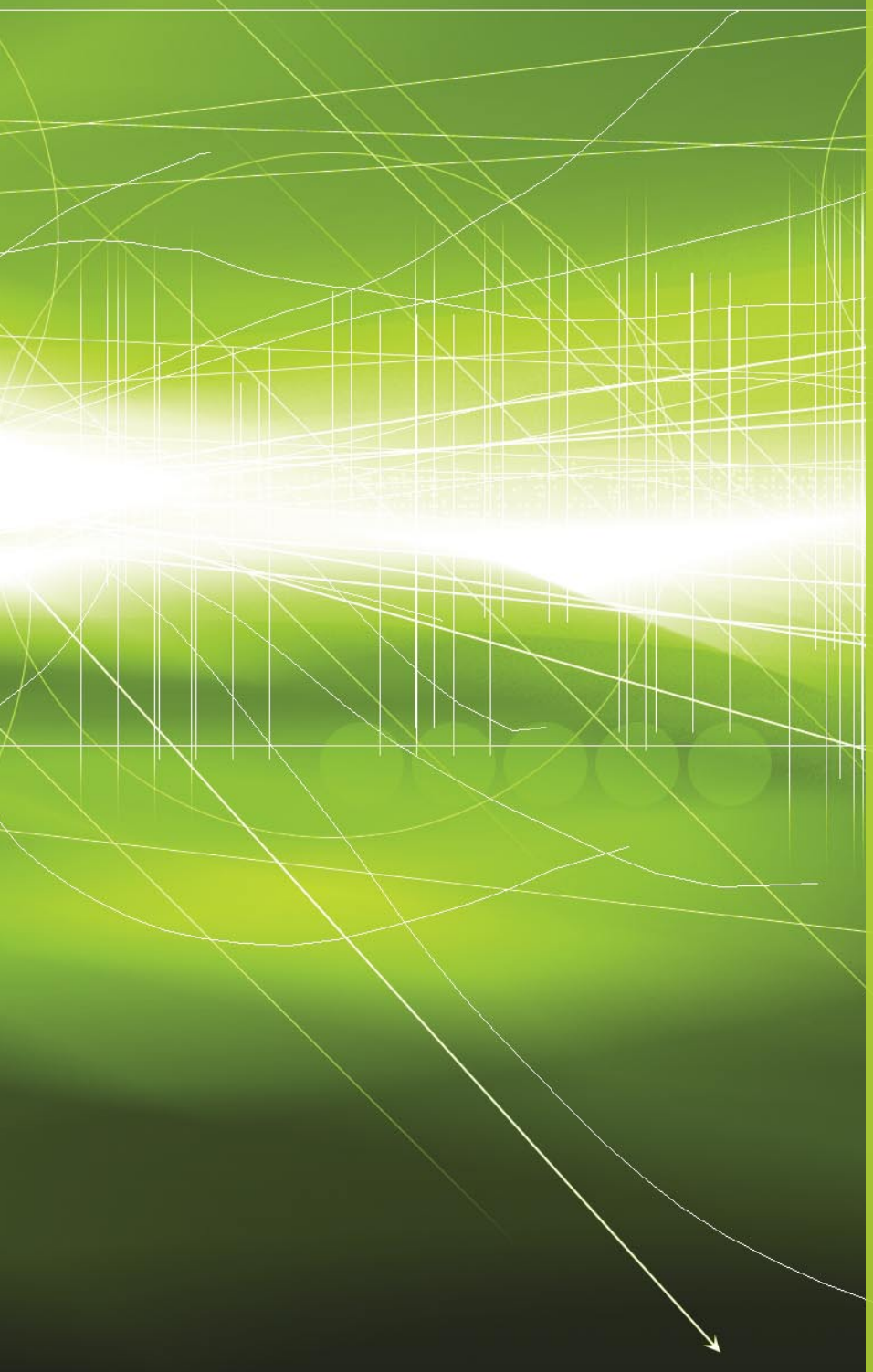

If you think VoIP is just an IP version of telecom-as-usual, think again. A host of applications are changing the phone call as we know it.

VoIP (voice over IP) technology is a rapidly expanding field. More and more VoIP components are being developed, while existing VoIP technology is being deployed at a rapid-and still increasing-pace. This growth is fueled by two goals: decreasing costs and increasing revenues.

Network and service providers see VoIP technology as a means of reducing their cost of offering existing voice-based services and new multimedia services. Service providers also view VoIP infrastructure as an economical base on which to build new revenue-generating services. As deployment of VoIP technology becomes widespread and part of a shared competitive landscape, this second goal will become more important, with service providers working to increase their market bases.

Most current and envisioned VoIP services are so-called converged services, integrating features and functions from multiple existing services. Often, features from conventional voice-based telephony services are combined with those found in data network services. For example, click-to-dial services allow users to control telephone calls from Web browsers running on their personal computers. Converged services may also provide users with new media integration. For example, multimedia conference services allow users to interact with each other through calls in which they exchange both audio and video information (i.e., new versions of videophones). 
processing not supported by network elements. This additional processing must be handled at call endpoints. The flow of information into and from an endpoint is through the voice channel itself, and therefore specialized controls must be built on audio controls (e.g., conversations with human operators, DTMF, or IVR). In figure 1, these endpoint application servers are represented by the call-center IVR server, which terminates voice connections and communicates via in-band signaling using DTMF or voice recognition.

VoIP technology provides richer, more flexible foundations for building communication services. IP networks support independent connections for signaling and media traffic. This decoupling of signal and bearer traffic eliminates interference between the information flows; in-band signaling is not required. Thus, communication with application servers is simplified.

In addition, IP network topology allows any node to act as a server. Therefore, multiple application servers and user endpoints-located in one or several service provider domains — can communicate via IP to participate in service support.

Finally, IP transport is provided by various underlying

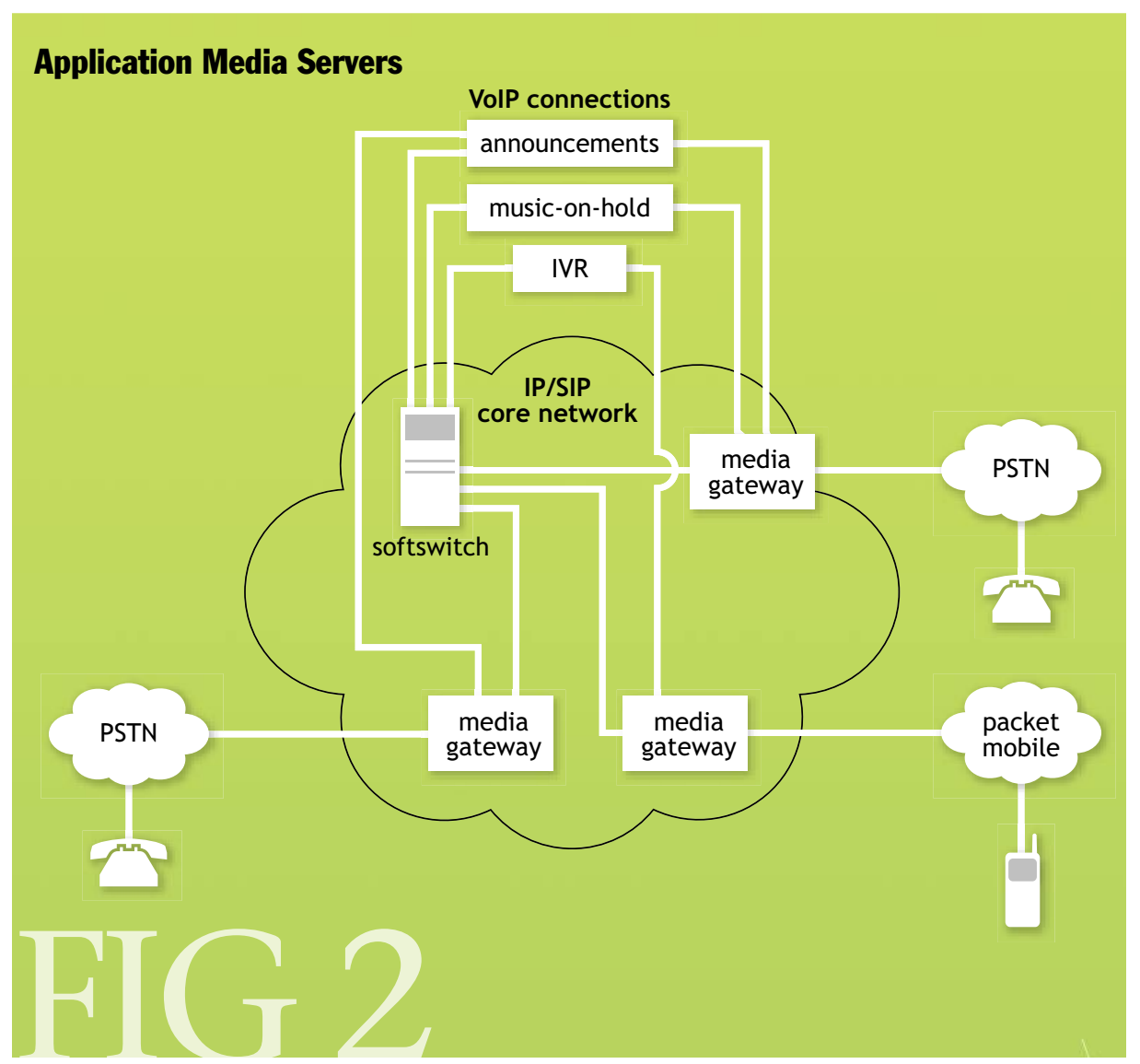

networks, and different network technologies can support different sets of services. For example, DSL and cable networks provide broadband IP connections that support realtime voice, data, and video services. Hence, these network providers can offer "triple-play" services to their customers.

Figure 2 shows how to implement a call-center service using VoIP technology. In the figure, user endpoints are telephones (not IP-based devices) attached to wireline or wireless access networks. An IP backbone interfaces to these specific access networks through border elements (e.g., media gateways). These gateways terminate voice calls for the users; they handle all TDM (time division multiplexing) voice traffic to and from users. The gateways recognize DTMF signals from the users and convert them to SIP (session initiation protocol) messages for the IP-based application servers. In addition, they convert between the users' TDM voice payload and RTP (realtime transport protocol) media packets, which are used by the media processors. Several IP-based application servers work in concert, coordinating their activities through SIP signaling to provide the call-center service. The softswitch contains a SIP proxy to support this SIP coordination, and it contains media control functions to support coordination of media processing. The application servers may be geographically distributed and separated from endpoints and switches. For example, Web sites can use stored voice or music files to provide announcements. They can act as music-on-hold servers; a single announcement server is not required.

VoIP technology provides a foundation for creating many new converged services through different combinations of components. For example, IVR and Web components can combine-using SIP as a common signaling protocol—-to create call-center services with access from Web browsers or IP phones, as well 
server must exchange agreed-upon protocols (typically including HTTP) so that Web pages can be transferred to the user. Also, the click-to-dial client and the click-to-dial server must exchange an agreed-upon protocol to request and control the required telephony functions.

Service coordination and composition become important issues in the development and execution of VoIP services, as multiple application servers are often involved. The industry must develop techniques to coordinate distinct service elements within sessions. One fundamental problem is that service behavior is difficult to describe both formally and conveniently, which makes service coordination labor-intensive. A related problem is that the multiple servers used to create a service might not be in the same network. Therefore, one service provider might not be willing to publish details of its server for another provider. Another difficulty is that services can interfere with each another. For example, if a conference participant temporarily leaves, generating music on hold, this behavior can interfere with or even block continuation of the conference by the remaining participants.

\section{NEW MEDIA INTEGRATION}

Many VoIP services are based on integration of multiple media. One such service is multimedia conferencing, which can be implemented by taking advantage of both SIP signaling and IP transport. SIP messages are available for server registration and rendezvous, as well as the controls that are needed to set up, conduct, and end sessions. Additional IP control messages are used to send mediaspecific commands. For example, service customers can use these commands to select video feeds, change codecs, change multicast groups, etc. IP transport is used to move the data representing the various media to and from servers and among users.

Figure 4 illustrates a conferencing service. Similar in overall structure to the IVR service depicted in figure 3 , this system is based on a different set of servers: a multimedia conference server, an audio bridge, video server, and data-sharing server. The conferencing server coordinates the activities of the data-specific servers, which manipulate different sets of packet data corresponding to appropriate media. For example, the audio bridge receives encoded voice from all participants and distributes combined voice data back to the participants. As the figure illustrates, uniformity of endpoint devices is not required-each customer can participant in a conference through a different type of endpoint-e.g., cellphone, analog phone, or laptop. The media transmitted to/from each participant depends upon the capabilities of the participant's endpoint device.

QoS (quality of service) is an important issue for IPbased multimedia services. Many current IP services have been deployed without QoS guarantees from underlying network providers. These services are successful because transport quality is sufficient to meet customer demands. Providers of these services, however, do not have assurances that their services can grow to meet the needs of larger customer bases while also meeting time constraints for the services. For example, IP-based voice and video services are being deployed in enterprises without explicit QoS support. Since the enterprise LANs used for transport

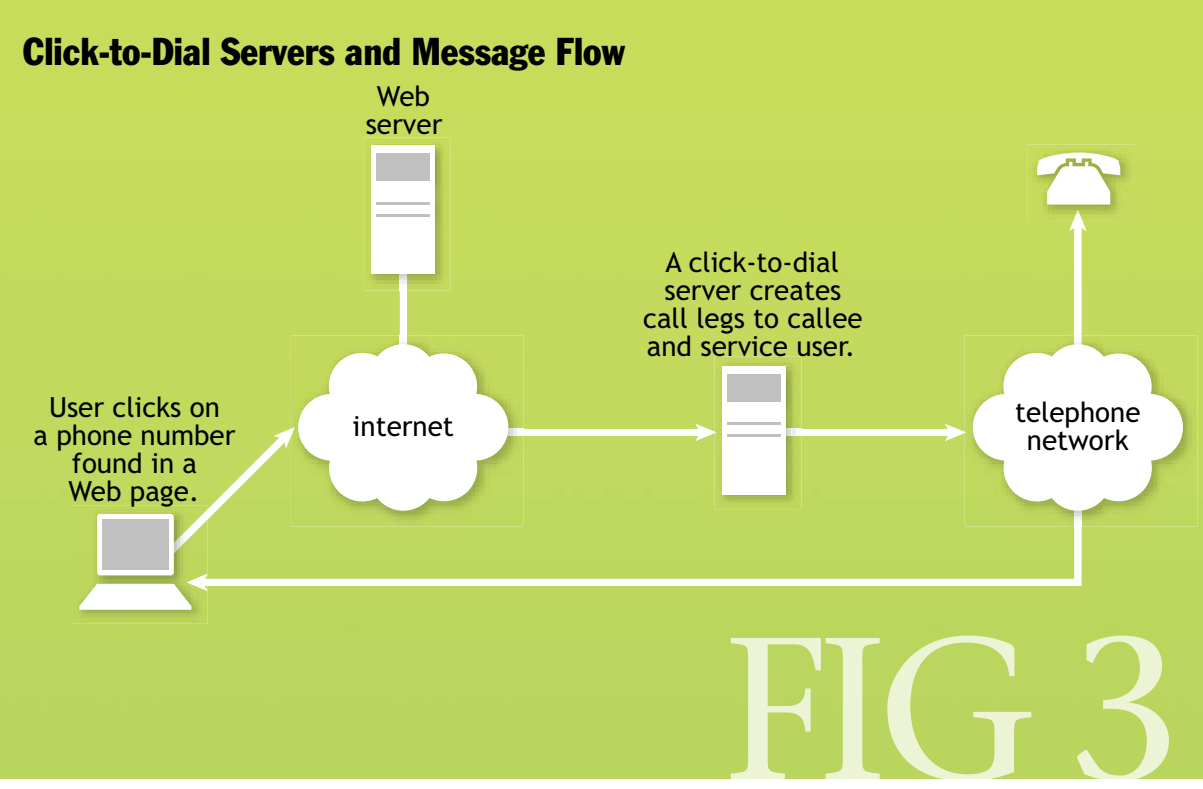

have enough bandwidth to allow over-provisioning for realtime voice, and video, these services are successful. Timely transport of time-sensitive data, however, to support realtime multimedia conversations across worldwide networks, is harder to ensure.

We must solve these problems by using adequate transport performance and servers within the signaling and media transport paths that can react to messages within realtime constraints. These servers must process both 


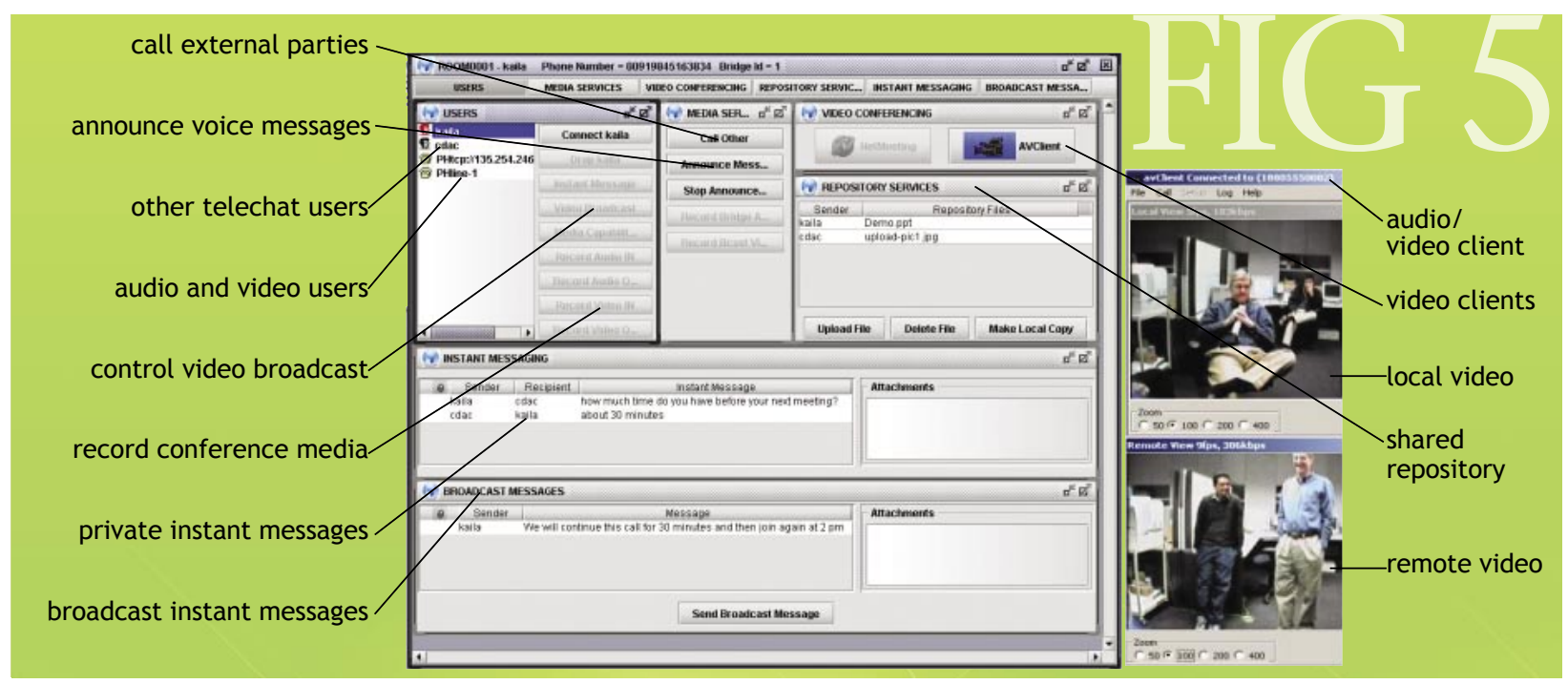

services offered on such networks, along with many data services. The same network will also support video services from videoconferencing to entertainment video.

More important, these services allow convergence at the control and user levels. A user can initiate a call or TV program from the Web and then send a video from a camera phone to the user's home Web site. Common Web-based services can be used for provisioning the user's personal choices. Clearly, this is only the beginning of exciting services offered by full multimedia on IP.

An important architectural change is that all application servers will move out of specific networks and become more access-independent. Networks will become multiservice platforms. To do this effectively, networks have to provide flexible QoS mechanisms and the ability to create virtual networks to match the services being deployed. This is where many of VoIP challenges remain to be solved. Specifically, we still need ways to specify network requirements of a particular application (e.g., multiparty audio-conferencing) and we need to be able to map that to the multiservice network. Finally, we need to be able to provision such services and monitor their execution to guarantee delivery.

Last, but not least, is the challenge of integrating the ever-smarter endpoint and endpoint-based applications with the network-centric view presented earlier. Besides new service interaction issues, this raises many new concerns about ownership of the user's data, authentication, billing for services, and responsibility for security.

VoIP is here and already leading the way not just to cheaper voice calls but also to a host of new applications. We need to focus on the challenges to enable a host of new multimedia applications. $Q$

\section{LOVE IT, HATE IT? LET US KNOW}

feedback@acmqueue.com or www.acmqueue.com/forums

SUDHIR AHUJA is vice president of the Converged Networks and Services Research Laboratory at Bell Labs/Lucent Technologies, where he is leading research in converged networks, services, speech recognition, text-to-speech coding techniques, video-based communication, and novel multimedia applications. He designed and developed the first large-scale multiprocessor at Bell Labs and championed the first Internet-based video conferencing system. His current interests are in the field of communication applications over the Internet.

Ahuja obtained his M.S. and Ph.D. degrees in electrical engineering from Rice University. His undergraduate education was at the Indian Institute of Technology, Bombay, where he received the President's Gold Medal for outstanding academic performance. He is a Fellow of Bell Labs and has served as chairman for the Multimedia Services and Terminals Committee of the IEEE Society, area editor for the IEEE Communications Committee, and editor for Transactions on Networking, a joint publication of IEEE and ACM. BOB ENSOR is a technical manager in the Services Infrastructure Research Department at Bell Labs/Lucent Technologies. He leads research and development efforts in next-generation network architectures and components. Earlier, he served as principal researcher in several projects at Bell Labs, including broadband service data centers, multimedia messaging systems, shared virtual worlds for the Internet, and multimedia conferencing systems. Ensor holds several patents and has published numerous papers. He received his Ph.D. in computer science from SUNY at Stony Brook. (c) 2004 ACM 1542-7730/04/0600 $\$ 5.00$ 